\title{
Methodology Proposal of ADHD Classification of Children Based on Cross Recurrence Plots
}

\section{Marco Aceves-Fernandez ( $\nabla$ marco.aceves@gmail.com )}

Universidad Autónoma de Querétaro: Universidad Autonoma de Queretaro https://orcid.org/00000002-5455-0329

\section{Original Research}

Keywords: Attention-Deficit Hyperactivity Disorder (ADHD), Electroencephalogram (EEG), CRP, CRQA, Recurrence Plots

Posted Date: February 2nd, 2021

DOl: https://doi.org/10.21203/rs.3.rs-163507/v1

License: (c) (1) This work is licensed under a Creative Commons Attribution 4.0 International License. Read Full License

Version of Record: A version of this preprint was published at Nonlinear Dynamics on March 10th, 2021. See the published version at https://doi.org/10.1007/s11071-021-06336-z. 


\title{
Methodology Proposal of ADHD Classification of Children based on Cross Recurrence Plots
}

\author{
M.A. Aceves-Fernandez* \\ Universidad Autónoma de Querétaro, Faculty of Engineering, Cerro de las Campanas S/N, 76010, Querétaro, \\ México. \\ * Author to whom correspondence should be addressed: marco.aceves@gmail.com*
}

\begin{abstract}
Dealing with electroencephalogram signals (EEG) are often not easy. The lack of predicability and complexity of such non-stationary, noisy and high dimensional signals is challenging. Cross Recurrence Plots (CRP) have been used extensively to deal with detecting subtle changes in signals even when the noise is embedded in the signal. In this contribution, a total of 121 children performed visual attention experiments and a proposed methodology using CRP and a Welch Power Spectral Distribution have been used to classify then between those who have ADHD and the control group. Additional tools were presented to determine to which extent the proposed methodology is able to classify accurately and avoid misclassifications, thus demonstrating that this methodology is feasible to classify EEG signals from subjects with ADHD. Lastly, the results were compared with a baseline machine learning method to prove experimentally that this methodology is consistent and the results repeatable.
\end{abstract}

Keywords: Attention-Deficit Hyperactivity Disorder (ADHD), Electroencephalogram (EEG), CRP, CRQA, Recurrence Plots.

\section{Introduction}

Electroencephalograph (EEG) signals have been investigated abundantly. It comes with many challenges (Subasi, 2005). For instance, the non-linear nature of such signals make it difficult to classify them using statistical and machine learning techniques. Some of the difficulties when trying to classify such signals are:

- Statistical approaches are often not accurate enough due to the highly non-linearity of the EEG signals.

- Some statistical and machine learning methods process the signal knowing there is an event (an attention event, in this case), but the method has problems locating the event itself.

- Surface EEG signals have a large amount of noise embedded in the signal, which may considerable increase the complexity of the classification.

- Most Artificial Intelligence (AI) and machine learning methods require extensive training for pattern recognition of the signals. 
To deal with these difficulties, a methodology is presented to classify EEG signals for both children with ADHD and Control groups using recurrence plots (RP). RP is a graphical representation of the amount of time at which two states of a system exist in the same phasespace neighborhood (Marwan, 2013). With these graphical representations, the dynamics of a highly lineal set of signals may be studied. (Aceves-Fernandez, 2014; Fernandez-Fraga, 2019).

Furthermore, since RP and Cross Recurrence Plots (CRP) are visual representation of the data, an analysis called RQA (recurrence quantification analysis) must be carried out in order to quantify the length and number of the recurrences, the phase trajectories, the system's entropy, among others (Elias, 2015; Silva, 2015)

\section{Background}

\subsection{EEG Signals}

The set of electroencephalogram (EEG) signals is composed of a series of electrical potentials that changes over time on different channels according to the international 10-20 standard (Fernandez-Fraga, 2019). An example of a typical output from the EEG is shown on figure 1.

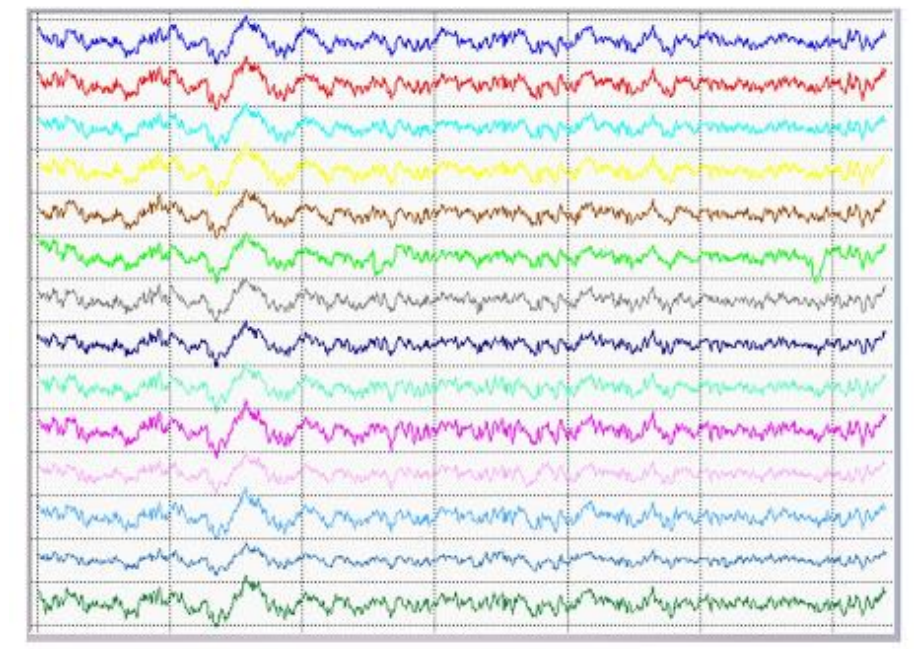

Figure 1. Set of signals derived from an electroencephalogram (EEG). 14 channels, sampling for $6.5 \mathrm{~s}$ (Fernandez-Fraga, 2019)

Many methods have been used for EEG signal classification. For instance, Zhou (2020) used a Radial Basis Function (RBF) Support Vector Machines to classify EEG signals, whiles Richhariya (2018) used another variation of an SVM to classify EEG. Also, Dose (2018) used mutual information to classify EEG signals with a Brain Computer Interface (BCI) and Satapathy (2017) used a popular swarm intelligence method called particle swarm optimization (PSO) for epilepsy identification using EEG signals. 
Furthermore, deep neural networks (DNN) have been used as well to classify EEG signals. For instance, Kumar (2016) used a EEG classification for motor imagery based on DNN and Nagabushanam (2019) used a recurrent network called LSTM to classify such signals.

\section{2. $\mathrm{ADHD}$}

According to some authors (Rau, 2020; Felt, 2014), attention-deficit/hyperactivity disorder (ADHD) is the most common behavioral disorder in children. For a correct diagnosis of ADHD in children, many factors must be considered. Some of them are: poor attention, distractibility, hyperactivity, impulsiveness, poor academic performance, or behavioral problems at home or at school (Rau, 2020; Felt, 2014).

There are some studies that used machine learning or artificial intelligence methods to classify ADHD data. For instance, Peng (2013) used ELM (Extreme Learning Machines) using MRI data, whilst Tenev used support vector machines (SVM) to classify ADHD in adults.

Also, Eslami (2018) used SVD (singular value decomposition) to classify ADHD patients based upon their dissimilarities and Mueller (2010) used independent components and a machine learning system to classify the patients. Furthermore, Sadarnezhad (2011) used linear discriminant analysis to detect features of ADHD in EEG signals, whilst Ghassemi (2012) used a similar approach to detect features on EEG signals.

Lastly, Kuang (2014) used deep neural network to attempt to classify ADHD subject using fMRI images.

\subsection{Cross Recurrence Plots}

Recurrence plots have been used largely for a number of applications. For instance, Aboofazeli (2018) have used RP and CRP for distinguishing features for breathe and swallowing sound. Also, Litak $(2011,2013)$ had used RPs and CRP to describe the features of the vibration for milling process, whilst Demos (2017) used RP to evaluate musical performance.

Also, Bastos (2011) has used RP to determine trend on stock markets, whilst Addo (2013) also used RP for financial applications. Furthermore, Rashvandi (2015) have been able to distinguish between different breath sounds using RQA and Nalband (2016) determined features to classify knee disorders using cross recurrence plots (CRP).

Finally, Villamor (2017) has used CRP to classify novice and expert programmers by tracking eye movements.

In terms of EEG signals with the use of RP, Khodabakhshi (2020) used RP for the analysis of emotions, Ngamga (2016) and Torse (2019) were able to classify patients that suffered epileptic seizures. Algo, Becker (2010) used RP strategies to monitor patients under anaesthesia. At present, the authors are unaware of any studies using RP or CRP for the classification of ADHD in children with similarities in comparison with the methodology presented in this contribution. 
A Cross Recurrence Plot (CRP) is a two-dimensional Figure that represents the occurrences between two different dynamic systems in an m-dimensional phase space. Cross recurrent matrix is defined by Eckmann (1987) as:

$$
\begin{gathered}
\mathrm{CR}_{i, j}=\Theta\left(\varepsilon-\left\|\vec{x}_{i}-\vec{y}_{j}\right\|\right), \\
i=1, \ldots, N, j=1, \ldots, M,
\end{gathered}
$$

Where:

$\vec{x}_{i}$ and $\vec{y}_{j}$ represent the trajectories in an m-dimensional space

$\Theta(\cdot)$ is the Heaviside function and;

$\|\cdot\|$ is the Euclidean norm

The RP has a main diagonal line, Since $\mathrm{CR}_{\mathrm{i}, \mathrm{i}}=1(\mathrm{i}=1 \ldots \mathrm{N})$, with the length depending on the largest Lyapunov exponents as explained by Aceves (2019).

In order to accurately calculate the recurrence quantification analysis features (RQA) for the problem at hand, the embedding parameter must be determined priorly. According to several authors (Bradley, 2002; Gao, 2000; Marwan 2004, 2007) the success or failure of RQA measures depend upon the correct calculation of the parameters which are: time delay, norm, recurrence threshold and embedding dimension.

Time delay is calculated since the noise is largely to increase as the dimension increases due to the non-linearity of the signal. Having a large time delay will not reconstruct accurately the signal in its original phase space. For that reason, the time delay was calculated using the method average mutual information and was set to 1 as shown on Fernandez-Fraga (2019).

The norm chosen in this contribution was calculated using Euclidean norm since it was demonstrated that this was the best type of norm to be used to this type or problem (March, 2005; Strozzi, 2007; Zou, 2010). Furthermore, the recurrence threshold must be as large as five times the standard deviation of the observational noise, i.e. $\varepsilon>5 \sigma$ (Goswami, 2012; Popescu, 2014), which in this case was set to 2.

Lastly, the embedding dimension was calculated using the False Nearest Neighbors algorithm as shown by Zou (2010), which in this case was calculated as $m=5$ using the Taken's theorem as demonstrated by Huke (2006).

It is important to define the measures to quantify the recurrence structures, called Cross Recurrence Quantification Analysis (CRQA). The measures considered in this contribution are: Recurrence Rate, Determinism, Entropy, Laminarity, Trapping Time and Trend (Ngamba, 2016; Marwan, 2013).

\subsubsection{Recurrence Rate}

The recurrence rate is a measure of density of the points or recurrences in the system (Gao, 2000). The recurrence rate is given by: 


$$
\mathrm{RR}=\frac{1}{N^{2}} \sum_{i, j}^{N} \mathbf{R}_{i, j}=\frac{1}{N^{2}} \sum_{l=1}^{N} I P(l),
$$

\subsubsection{Determinism}

The Determinism (DET) corresponds to the local predictability of a system. Determinism also measures the discrepancies of the diagonal lines (Marwan, 2013). The Determinism of a system is calculated as:

$$
D E T=\frac{\sum_{l \geq l_{\min }} l P(l)}{\sum_{i, j} R_{i, j}}
$$

\subsubsection{Entropy}

This measure refers to the Shannon entropy of the frequency distribution of the diagonal line lengths, this is, the variability in the lengths of the diagonal lines (Yulmetyev, 1999).

The entropy of a system is given by:

$$
E N T=-\sum_{l=l_{\min }}^{N} p(l) \log p(l) \operatorname{withp}(l)=\frac{p^{\varepsilon}(l)}{\sum_{l=l_{\min }}^{N} P^{\varepsilon}(l)}
$$

\subsubsection{Laminarity}

The Laminarity (LAM) may be defined as the frequency distribution of the lengths that form vertical lines (Marwan, 2007). Laminarity is also the evidence of the chaotic transitions and is related to the number of laminar phases in the system, which is represented by the occurrence of vertical lines in the recurrence plot

$$
L A M=\frac{\sum_{V=v_{\min }}^{N} v \cdot P(v)}{\sum_{V=1}^{N} v \cdot P(v)}
$$

\subsubsection{Trapping Time}

Trapping Time measures the difference of thee length of vertical lines (6):

$$
T T=\frac{\sum_{v=v_{\min }}^{N} P P(v)}{\sum_{v=v_{\min }}^{N} P(v)}
$$


where $\mathrm{v}$ is the length of the vertical lines, $\mathrm{v}_{\min }$ is the shortest length that is considered a line segment and $\mathrm{P}(\mathrm{v})$ is the distribution of the corresponding lengths.

\subsubsection{Trend}

The trend is a linear regression coefficient over the recurrence point density of the diagonals parallel to the Line Of Identity (LOI). The trend measurement is given by:

$$
\operatorname{TREND}=\frac{\sum_{i=1}^{N}\left(i-\frac{N}{2}\right)\left(R R_{i}=\left\{R R_{i}\right\}\right)}{\sum_{i-1}^{N}\left(i=\frac{N}{2}\right)^{2}}
$$

\section{Materials and Methods}

\subsection{Materials}

A total of one hundred and twenty one children were selected. The EEG reading protocol was based on the 10-20 system using 19 channels (Fz, Cz, Pz, C3, T3, C4, T4, Fp1, Fp2, F3, F4, F7, F8, P3, P4, T5, T6, O1 and O2) and 2 reference electrodes (Fernandez-Fraga, 2019). The electrodes sent information with a $128 \mathrm{hz}$ sampling frequency.

In this context, 61 children were diagnosed with ADHD and 60 healthy controls, boys and girls aged 7-12 years old. The ADHD children were diagnosed by experienced psychiatrist using DSM-IV criteria (Mohammadi, 2016).

As shown by Samavati (2012), the Children were shown with pictures that may be attractive for them to watch (e.g. cartoons) and then asked how many characters they saw.

\subsection{Proposed Methodology}

The proposed methodology follows these steps:

a) The signals were acquired for each electrode and each subject (both ADHD and control groups) as explained by Mohammadi (2016)

b) The relevant electrodes must be selected. In this case, an experiment was performed to determine whether the accuracy decreases for certain electrodes or is region or electrode dependent.

c) If the signals contained readable information, they will be normalized using a $\mathrm{z}$-score method, otherwise they will be discarded.

d) Once the signals were normalized, the recurrence features must be selected.

To ensure repeatability and consistency, the embedded dimension $\mathrm{m}=5$, delay $=1$ by mutual information method and Euclidean norm were fixed.

e) The recurrence Quantification parameters were calculated for each electrode of each subject (both ADHD and Control groups) unless th signal was discarded as explained on step c. The recurrence parameters calculated were: recurrence rate (Rec), Determinism (Det), Entropy (Ent), Laminarity (Lam), Trapping Time (TT) and Trend.

f) Power Spectral Density (PSD) must be calculated for each electrode and each subject using the Welch method (Welch, 1967; Barbe, 2009). 
g) Once RQA parameters were calculated, interpretation of the results must be performed.

The methodology described in the present section may be graphically shown on Figure 2 .

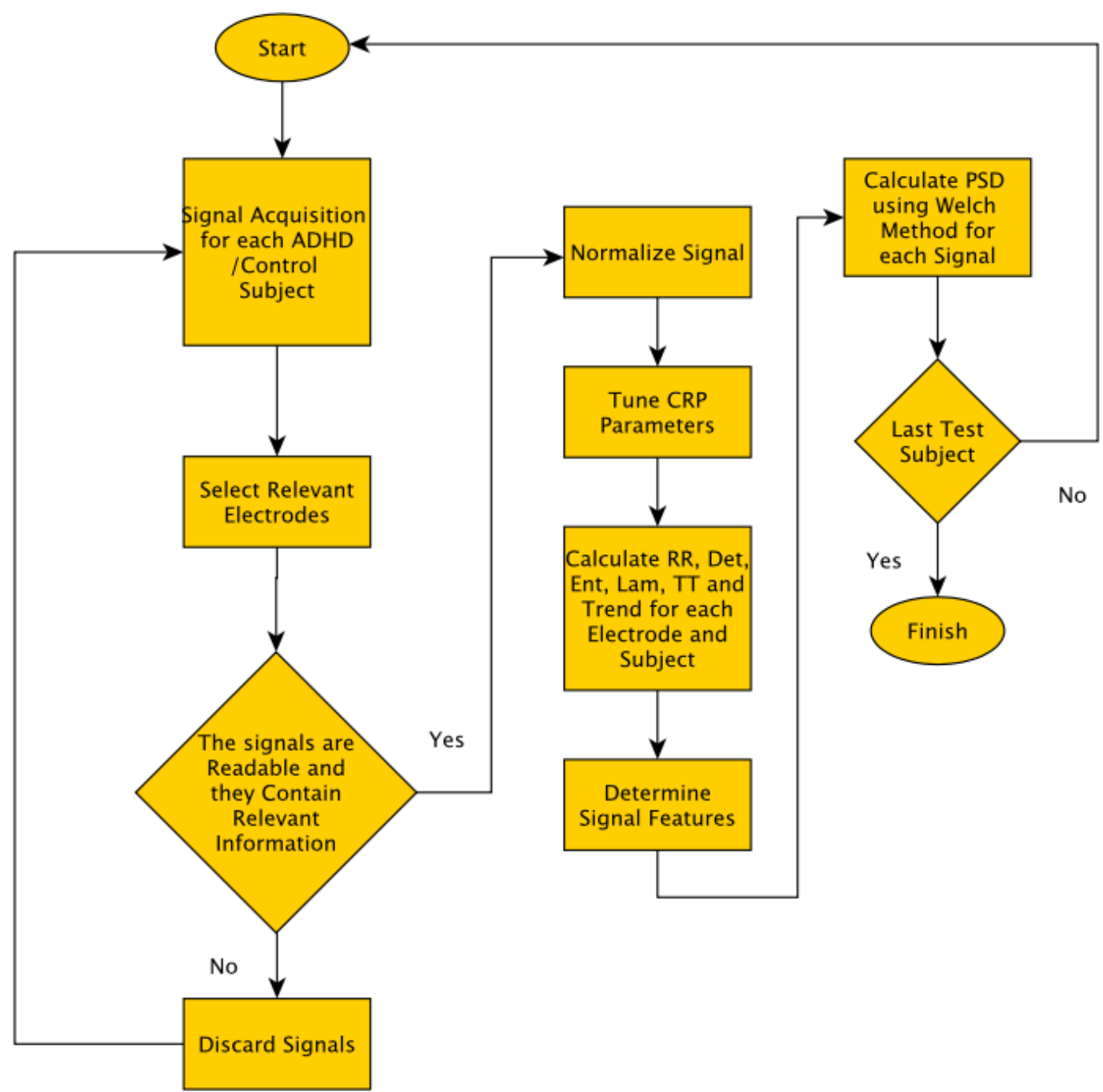

Figure 2. Methodology used in the present work.

\section{Experimental Results}

The interpretation of these non-stationary, highly dimensional, nonlinear EEG signals is often not straightforward. In this contribution, the Welch method is used to determine the segments for power spectral density called periodograms as explained by the Welch method (Welch, 1967; Rahi, 2014) for each electrode and each RQA feature.

Figure 3a shows the recurrence rate for all test for each electrode for ADHD group using the Welch method, whilst Figure $3 \mathrm{~b}$ the recurrence rate for the control group. In both figures it shows the difference between the recurrence rate for the ADHD and control groups. This is specially so in frequencies greater than $0.5 \mathrm{rad} / \mathrm{sample}$. Also, figures $3 \mathrm{c}$ and $3 \mathrm{~d}$ show the determinism for ADHD and control groups, respectively. In these figures, the difference 
between the frequencies for both groups starts be be evident from $0.5 \mathrm{rad} / \mathrm{sample}$ onwards. Furthermore, Figures 3e and 3f show the RQA Entropy calculated for both ADHD and control groups. Both figures show similar behavior in which from $0.6 \mathrm{rad} / \mathrm{sample}$ both groups show differences in their frequencies.

In terms of Laminarity (Figures $3 g$ and $3 h$ ) it could be noted that for all frequencies, the signals for the ADHD group seem more similar with respect with the control group. Finally both Trapping Time (Figures $3 \mathrm{i}$ and $3 \mathrm{j}$ ) and Trend (Figures 3k and 31), show similar values for frequencies smaller than $0.7 \mathrm{rad} / \mathrm{sample}$. For most cases, the greater differences between ADHD and control groups are shown as the frequencies increase.

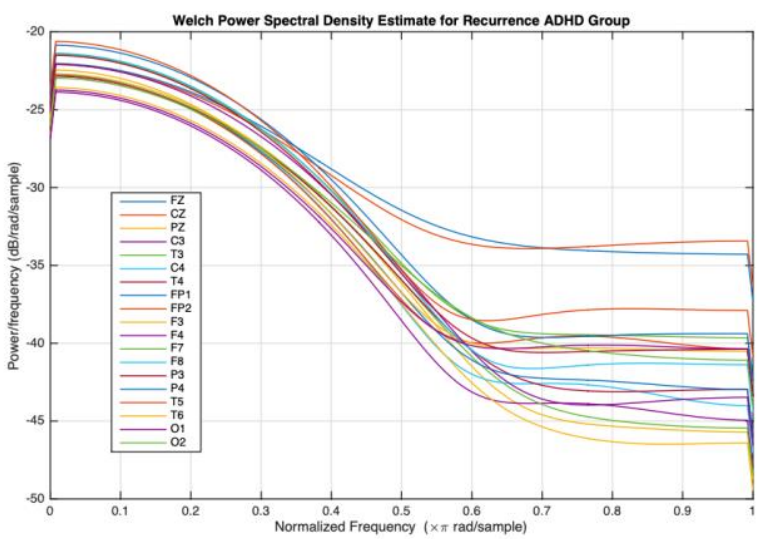

(a)

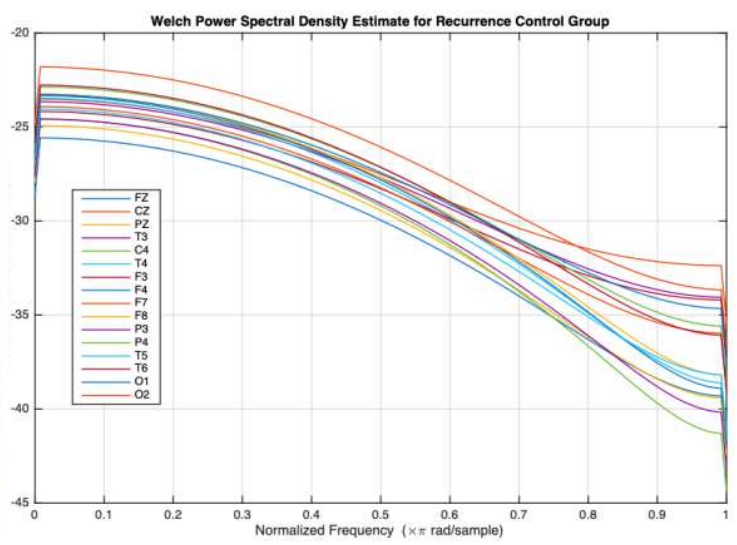

(b)

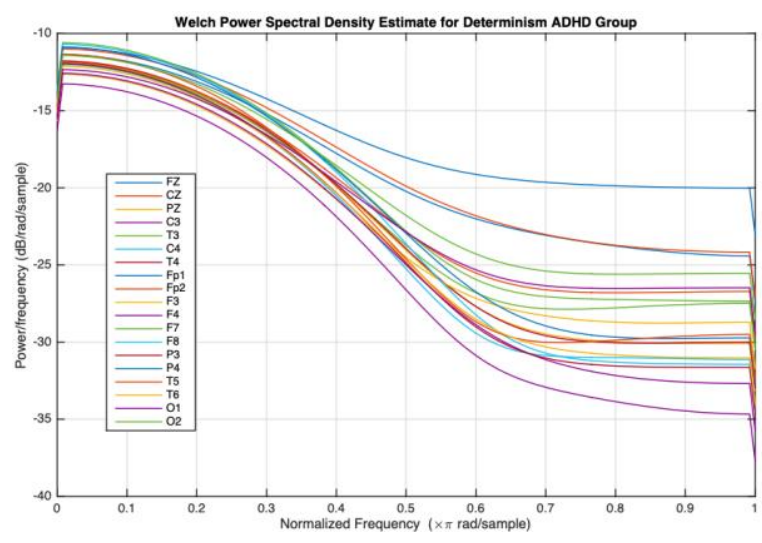

(c)

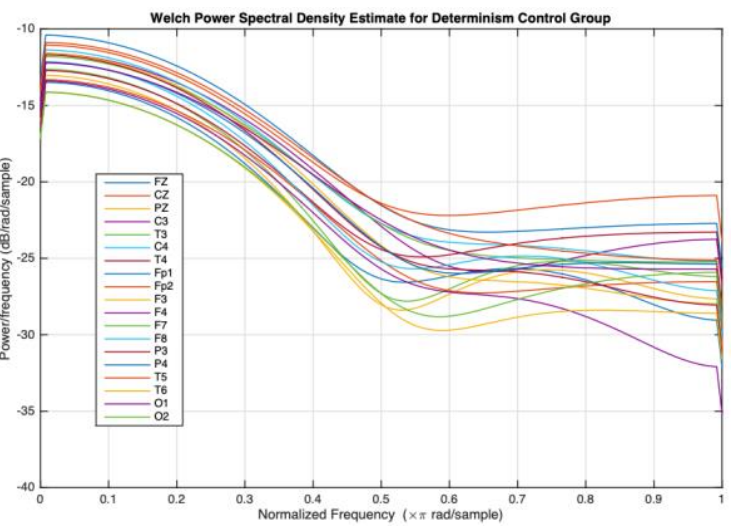

(d) 


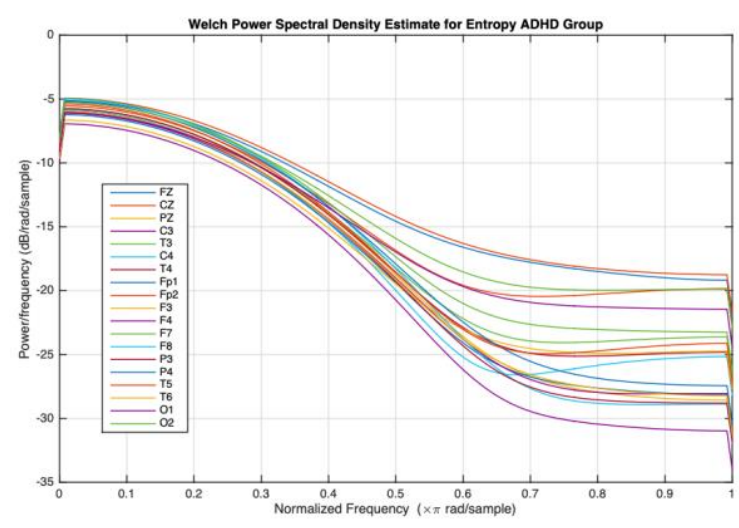

(e)

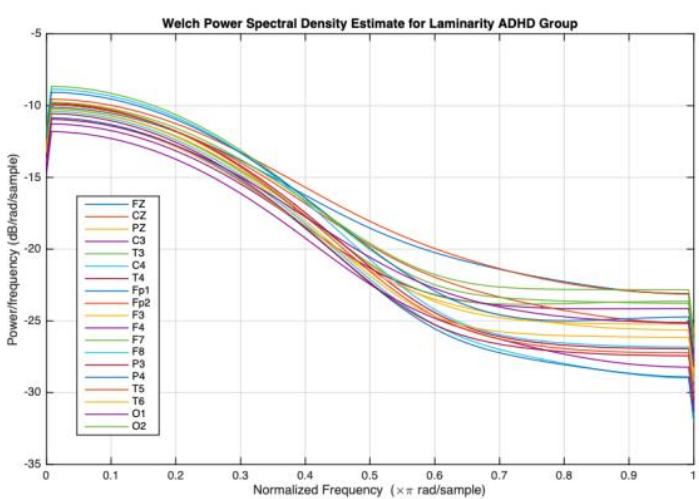

(g)

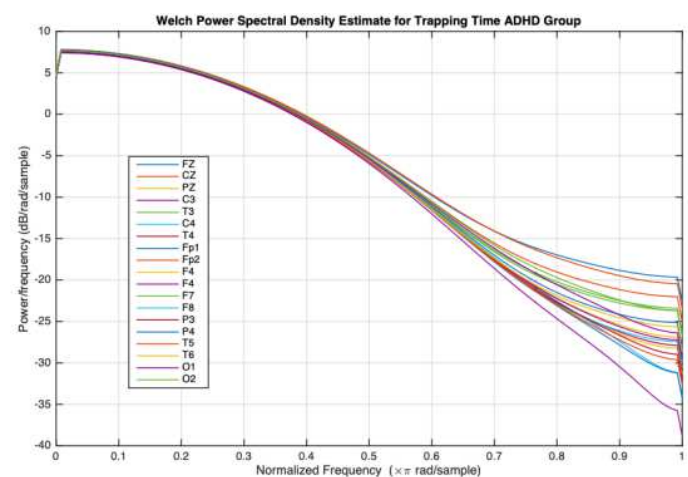

(i)

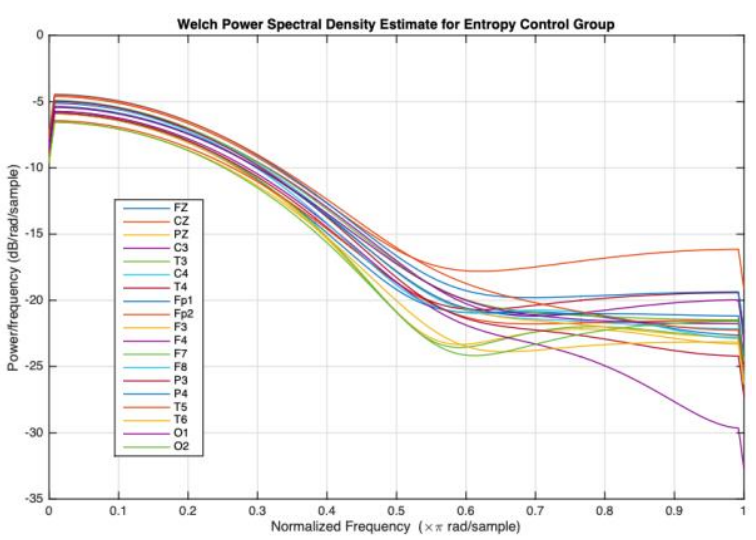

(f)

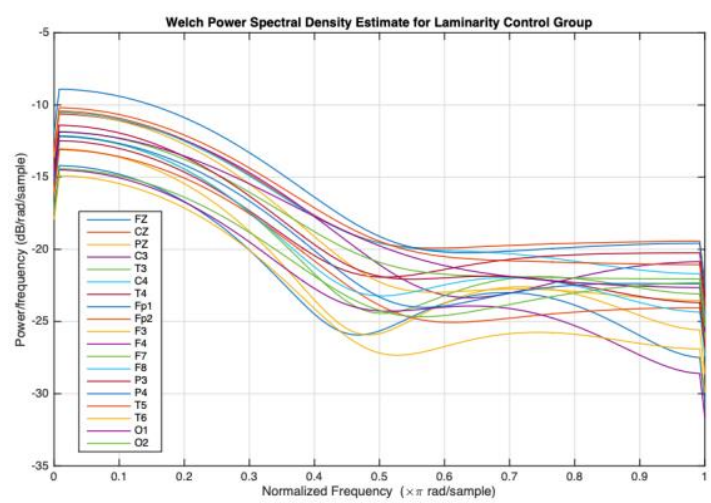

(h)

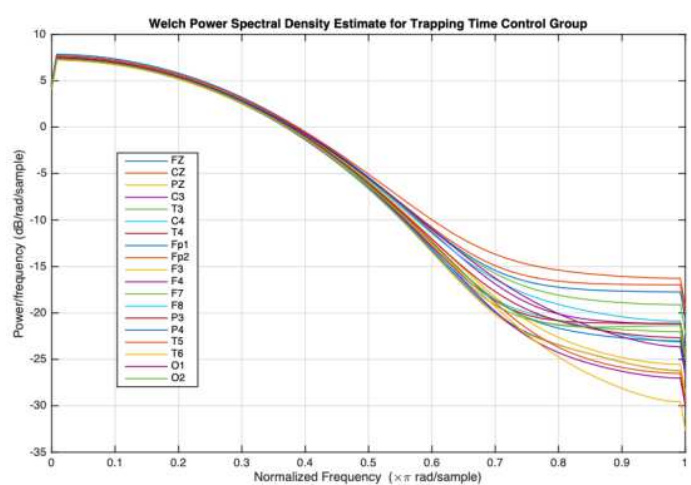



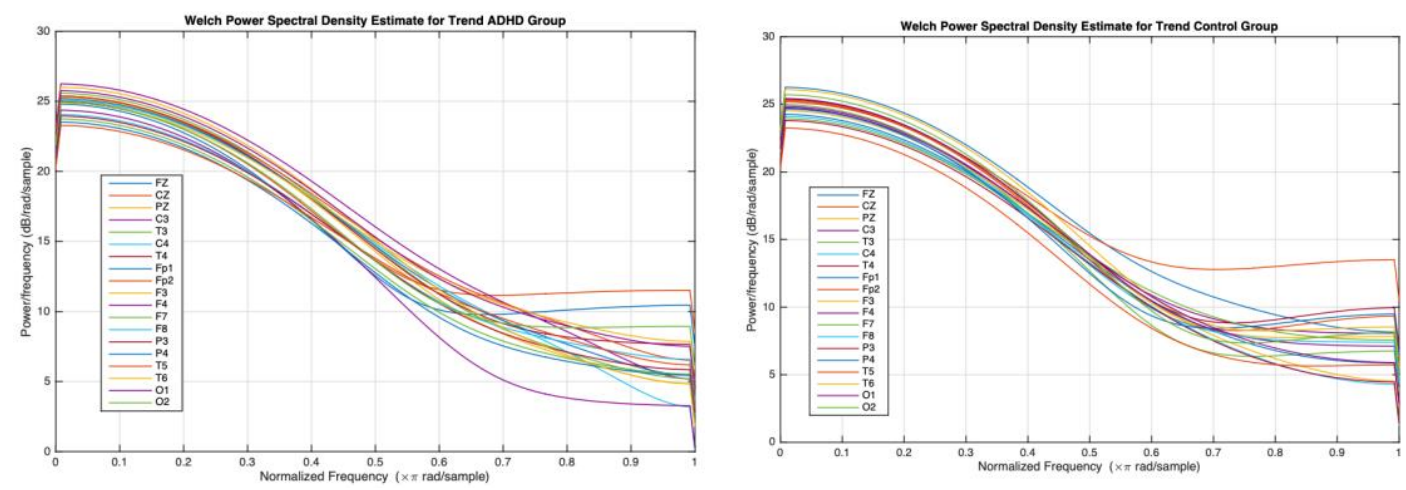

(k)

(1)

Figure 3. Results of RQA Measures using the Welch Power Spectral Density Method. a)

Recurrence rate vs Power Frequency for ADHD Group, b) Recurrence rate vs Power Frequency for Control Group, c) Determinism vs Power Frequency for ADHD Group, d) Determinism vs Power Frequency for Control Group, e) Entropy vs Power Frequency for ADHD Group, f) Entropy vs Power Frequency for Control Group g) Laminarity vs Power Frequency for ADHD Group, h) Laminarity vs Power Frequency for Control Group, i) Trapping Time vs Power Frequency for ADHD Group, j) Trapping Time vs Power Frequency for Control Group, k) Trend vs Power Frequency for ADHD Group and 1) Trend vs Power Frequency for Control Group.

As shown on Figure 3, there are significant differences between the ADHD and control groups in terms of the power frequency for each electrode. However, the visual differences do not quantify the extend to which the proposed methodology is feasible to classify both groups. Hence, accuracy (AC), sensitivity (SE) and specificity (SP) were calculated for each RQA metric and experiments were made to ensure that this methodology is consistent. A total of 1000 experiments were performed using all the data available for each electrode using the 80-20 method. This is, in each experiment, $80 \%$ of the data is used as the model whereas the remaining $20 \%$ is used for testing. For each correct detection, a true positive (TP) and true negative (TN) are 
given whereas a mis-detection is given by false positive (FP) and false negative (FN). AC, SE and SP are calculated as follows:

$$
\begin{aligned}
\text { Sensitivity } & =\frac{T P}{T P+F N} \\
\text { Specificity } & =\frac{T N}{T N+F P} \\
\text { Accuracy } & =\frac{T P+T N}{T N+T P+F N+F P}
\end{aligned}
$$

Table 1 shows the AC, SE and SP of recurrence rate for each EEG electrode. In this table, it is shown that specificity is reliable ranging from 0.9724 for electrode $\mathrm{Cz}$ to 0.9201 for electrode $\mathrm{P} 4$ with a mean of 0.949 . This seems to indicate that regardless of the experiment, and the data used as testing, most of the electrodes show high accuracy. In terms of SP, it shows a mean of 0.9461 with a higher value of SP of 0.9663 for electrode O1. This seems to indicate that for recurrence rate, the results are not prone to mis-calculations of false positives and false negatives. Lastly, high values of AC are also shown, ranging from 0.9144 to 0.9675 with a mean of 0.9493 .

\begin{tabular}{|l|c|c|c|c|c|c|c|c|c|c}
\hline Recurrence & $\underline{\mathrm{Fz}}$ & $\underline{\mathrm{Cz}}$ & $\underline{\mathrm{Pz}}$ & $\underline{\mathrm{C} 3}$ & $\underline{\mathrm{T} 3}$ & $\underline{\mathrm{C} 4}$ & $\underline{\mathrm{T} 4}$ & $\underline{\mathrm{Fp} 1}$ & $\underline{\mathrm{Fp} 2}$ & $\underline{\mathrm{F} 3}$ \\
\hline Sensitivity (SE) & 0.9621 & 0.9724 & 0.9602 & 0.9631 & 0.9445 & 0.9472 & 0.9201 & 0.9593 & 0.9551 & 0.9529 \\
\hline Specificity (SP) & 0.9537 & 0.9636 & 0.955 & 0.9462 & 0.9428 & 0.9439 & 0.9177 & 0.9416 & 0.9582 & 0.9593 \\
\hline Accuracy (ACC) & 0.9602 & 0.9664 & 0.9581 & 0.9577 & 0.9434 & 0.9465 & 0.9144 & 0.9491 & 0.9558 & 0.9613 \\
\hline & $\underline{\mathrm{F} 4}$ & $\underline{\mathrm{F} 7}$ & $\underline{\mathrm{F} 8}$ & $\underline{\mathrm{P} 3}$ & $\underline{\mathrm{P} 4}$ & $\underline{\mathrm{T} 5}$ & $\underline{\mathrm{T}}$ & $\underline{01}$ & $\underline{\mathrm{O} 2}$ & $\underline{\mathrm{Mean}}$ \\
\hline Sensitivity (SE) & 0.9552 & $\underline{0.9471}$ & $\underline{0.9666}$ & $\underline{0.9276}$ & $\underline{0.9201}$ & $\underline{0.922}$ & 0.9331 & $\underline{0.969}$ & $\underline{0.9592}$ & $\underline{0.94906}$ \\
\hline Specificity (SP) & 0.9586 & 0.941 & 0.9634 & 0.9277 & 0.9214 & 0.9294 & 0.9375 & 0.9663 & 0.9512 & 0.94613 \\
\hline Accuracy (ACC) & 0.9612 & 0.953 & 0.9675 & 0.9249 & 0.926 & 0.9275 & 0.9446 & 0.9635 & 0.9577 & 0.94931 \\
\hline
\end{tabular}

Table 1. Sensitivity, Specificity and Accuracy metrics for CRQA Recurrence Rate for the experiments performed for both groups.

Table 2 shows the SE, SP and AC of determinism for the experiments performed. In this table, is shown that the sensitivity from determinism ranges from 0.9306 to 0.9669 with a mean of 0.9497. In comparison, the baseline results given by Mohammadi (2016), which were 0.9228 using an MLP (Multi Layer Perceptron) are higher for every electrode in the results showed in this contribution. In terms of the specificity, determinism also shows high values for all electrodes, ranging from 0.9412 (P3 electrode) to $0.9651(\mathrm{Cz})$, which also seems to indicate that the methodology proposed detects false positives and false negatives. Finally, accuracy for RQA determinism ranges from 0.9372 (P4 electrode) to 0.9613 (both for Fz and F7 electrodes). This seems to indicate that the results are reliable every experiment performed. 


\begin{tabular}{|l|c|c|c|c|c|c|c|c|c|c|}
\hline Determinism & $\underline{\mathrm{Fz}}$ & $\underline{\mathrm{Cz}}$ & $\underline{\mathrm{Pz}}$ & $\underline{\mathrm{C} 3}$ & $\underline{\mathrm{T} 3}$ & $\underline{\mathrm{C} 4}$ & $\underline{\mathrm{T} 4}$ & $\underline{\mathrm{Fp} 1}$ & $\underline{\mathrm{Fp} 2}$ & $\underline{\mathrm{F} 3}$ \\
\hline Sensitivity (SE) & 0.9588 & 0.9606 & 0.9557 & 0.964 & 0.9421 & 0.9502 & 0.9384 & 0.9553 & 0.9516 & 0.9487 \\
\hline Specificity (SP) & 0.9572 & 0.9651 & 0.9511 & 0.9619 & 0.9466 & 0.9571 & 0.9415 & 0.9489 & 0.9507 & 0.9518 \\
\hline Accuracy (ACC) & 0.9613 & 0.9592 & 0.9582 & 0.9575 & 0.952 & 0.9444 & 0.939 & 0.9503 & 0.9536 & 0.9505 \\
\hline & $\underline{\mathrm{F} 4}$ & $\underline{\mathrm{F} 7}$ & $\underline{\mathrm{F}}$ & $\underline{\mathrm{P} 3}$ & $\underline{\mathrm{P} 4}$ & $\underline{\mathrm{T} 5}$ & $\underline{\mathrm{T}}$ & $\underline{\mathrm{O}}$ & $\underline{\mathrm{O}}$ & $\underline{\mathrm{Mean}}$ \\
\hline Sensitivity (SE) & 0.9559 & $\underline{0.9669}$ & $\underline{0.9591}$ & $\underline{0.9343}$ & $\underline{0.9306}$ & $\underline{0.9491}$ & $\underline{0.9313}$ & $\underline{0.9447}$ & $\underline{0.9505}$ & $\underline{0.94974}$ \\
\hline Specificity (SP) & 0.9533 & 0.9635 & 0.9632 & 0.9412 & 0.9415 & 0.9476 & 0.939 & 0.9424 & 0.9532 & 0.95131 \\
\hline Accuracy (ACC) & 0.9571 & 0.9613 & 0.9667 & 0.9468 & 0.9372 & 0.9436 & 0.941 & 0.9485 & 0.9475 & 0.95128
\end{tabular}

Table 2. Sensitivity, Specificity and Accuracy metrics for CRQA Determinism for the experiments performed for both groups.

Table 3 shows the SE, SP and AC for entropy for the experiments carried out. In this table, is shown that sensitivity is slightly lower than recurrence rate and determinism. This ranges from 0.9035 (T4 electrode) to 0.9431 (F7 electrode) with a mean of 0.928 . Also, specificity shows results that ranges from 0.9112 (T4 electrode) to 0.9441 (F8 electrode) with a mean of 0.9289 . Furthermore, accuracy for entropy ranges from 0.9025 (T5 electrode) to 0.9381 (Fp2 electrode) with a mean of 0.9246 . It is noteworthy that the mean for all three metrics are fairly consistent and show a slightly higher accuracy to the baseline to which this work is been compared as shown on Mohammadi (2016) which is 0.9228 .

\begin{tabular}{l|c|c|c|c|c|c|c|c|c|c}
\hline Entropy & $\underline{\mathrm{Fz}}$ & $\underline{\mathrm{Cz}}$ & $\underline{\mathrm{Pz}}$ & $\underline{\mathrm{C} 3}$ & $\underline{\mathrm{T} 3}$ & $\underline{\mathrm{C} 4}$ & $\underline{\mathrm{T} 4}$ & $\underline{\mathrm{Fp} 1}$ & $\underline{\mathrm{Fp} 2}$ & $\underline{\mathrm{F} 3}$ \\
\hline Sensitivity (SE) & 0.9312 & 0.9365 & 0.9246 & 0.927 & 0.9112 & 0.9317 & 0.9035 & 0.9306 & 0.9337 & 0.9305 \\
\hline Specificity (SP) & 0.9336 & 0.9352 & 0.9313 & 0.9254 & 0.9164 & 0.9368 & 0.9112 & 0.9287 & 0.936 & 0.9211 \\
\hline Accuracy (ACC) & 0.932 & 0.9289 & 0.9277 & 0.9218 & 0.9175 & 0.9313 & 0.9144 & 0.9325 & 0.9381 & 0.9276 \\
& $\underline{\mathrm{F} 4}$ & $\underline{\mathrm{F}}$ & $\underline{\mathrm{F}}$ & $\underline{\mathrm{P}}$ & $\underline{\mathrm{P} 4}$ & $\underline{\mathrm{T}}$ & $\underline{\mathrm{T}}$ & $\underline{\mathrm{O}}$ & $\underline{\mathrm{O}}$ & $\underline{\mathrm{Mean}}$ \\
\hline Sensitivity (SE) & 0.9412 & 0.9431 & 0.9403 & 0.9221 & 0.9176 & 0.9114 & 0.92 & 0.9365 & 0.9377 & 0.92802 \\
Specificity (SP) & 0.944 & 0.9387 & 0.9441 & 0.9115 & 0.913 & 0.936 & 0.9212 & 0.9338 & 0.9312 & 0.92898 \\
\hline Accuracy (ACC) & 0.9297 & 0.932 & 0.9288 & 0.9131 & 0.9025 & 0.9229 & 0.9115 & 0.9303 & 0.9289 & 0.92468 \\
\hline
\end{tabular}

Table 3. Sensitivity, Specificity and Accuracy metrics for CRQA Entropy for the experiments performed for both groups.

Table 4 shows the SE, SP and AC for laminarity for the experiments. In this table, is shown that all metrics show a high value of true positives and true negatives, which seems to indicate that laminarity is a good RQA metric to classify ADHD from control group. More specifically, sensitivity ranges from 0.9666 (Fp1 electrode) with the lowest value of 0.938 (T6 electrode), which is not a low value by any means. In comparison, the baseline value is 0.9228 which is still higher than the lowest value of sensitivity for these experiments. Also, the experimental results for specificity show that it reaches a mean of 0.9529 , whereas the mean for accuracy is 0.9527 which is seems to indicate that laminarity is a reliable tool to classify ADHD 


\begin{tabular}{|l|c|c|c|c|c|c|c|c|c|c|}
\hline Laminarity & $\underline{\mathrm{Fz}}$ & $\underline{\mathrm{Cz}}$ & $\underline{\mathrm{Pz}}$ & $\underline{\mathrm{C} 3}$ & $\underline{\mathrm{T} 3}$ & $\underline{\mathrm{C} 4}$ & $\underline{\mathrm{T} 4}$ & $\underline{\mathrm{Fp} 1}$ & $\underline{\mathrm{Fp} 2}$ & $\underline{\mathrm{F} 3}$ \\
\hline Sensitivity (SE) & 0.9552 & 0.9601 & 0.9549 & 0.9583 & 0.9487 & 0.9442 & 0.9612 & 0.9666 & 0.9637 & 0.9512 \\
\hline Specificity (SP) & 0.9576 & 0.9557 & 0.9574 & 0.9588 & 0.9551 & 0.9503 & 0.9556 & 0.9612 & 0.9578 & 0.9595 \\
\hline Accuracy (ACC) & 0.9531 & 0.9573 & 0.9586 & 0.9564 & 0.9515 & 0.9474 & 0.966 & 0.9653 & 0.9551 & 0.9546 \\
& $\underline{\mathrm{F} 4}$ & $\underline{\mathrm{F} 7}$ & $\underline{\mathrm{F}}$ & $\underline{\mathrm{P} 3}$ & $\underline{\mathrm{P4}}$ & $\underline{\mathrm{T} 5}$ & $\underline{\mathrm{T}}$ & $\underline{\mathrm{O1}}$ & $\underline{\mathrm{O} 2}$ & $\underline{\mathrm{Mean}}$ \\
\hline Sensitivity (SE) & 0.961 & $\underline{0.9634}$ & $\underline{0.9576}$ & $\underline{0.9442}$ & 0.9475 & 0.9392 & $\underline{0.938}$ & $\underline{0.9487}$ & $\underline{0.9494}$ & $\underline{0.95315}$ \\
\hline Specificity (SP) & 0.9632 & 0.9567 & 0.9589 & 0.9403 & 0.9441 & 0.9415 & 0.9424 & 0.9521 & 0.9416 & 0.95294 \\
\hline Accuracy (ACC) & 0.9578 & 0.9583 & 0.9591 & 0.9417 & 0.9368 & 0.9433 & 0.9446 & 0.9482 & 0.9503 & 0.95272 \\
\hline
\end{tabular}

Table 4. Sensitivity, Specificity and Accuracy metrics for CRQA Laminarity for the experiments performed for both groups.

Table 5 shows SE, SP and AC for trapping time. In this table, it is shown that the sensitivity is also higher than the baseline work ranging from 0.9379 (T4) to 0.9632 (O1) with a mean of 0.9511 . Also, specificity shows a mean of 0.953 and accuracy a mean of 0.954 , which seems to indicate consistency between metrics for laminarity and a high probability of classification between ADHD and control groups.

\begin{tabular}{l|c|c|c|c|c|c|c|c|c|c}
\hline Trapping Time & $\underline{\mathrm{Fz}}$ & $\underline{\mathrm{Cz}}$ & $\underline{\mathrm{Pz}}$ & $\underline{\mathrm{C} 3}$ & $\underline{\mathrm{T} 3}$ & $\underline{\mathrm{C} 4}$ & $\underline{\mathrm{T} 4}$ & $\underline{\mathrm{Fp} 1}$ & $\underline{\mathrm{Fp} 2}$ & $\underline{\mathrm{F} 3}$ \\
\hline Sensitivity (SE) & 0.9535 & 0.9602 & 0.9581 & 0.9488 & 0.9402 & 0.9422 & 0.9379 & 0.9552 & 0.9559 & 0.9485 \\
\hline Specificity (SP) & 0.957 & 0.9623 & 0.9577 & 0.9531 & 0.9433 & 0.9441 & 0.9447 & 0.9512 & 0.9522 & 0.9444 \\
\hline Accuracy (ACC) & 0.9581 & 0.9656 & 0.944 & 0.9557 & 0.9519 & 0.9403 & 0.9511 & 0.956 & 0.9567 & 0.9516 \\
\hline & $\underline{\mathrm{F} 4}$ & $\underline{\mathrm{F} 7}$ & $\underline{\mathrm{F} 8}$ & $\underline{\mathrm{P} 3}$ & $\underline{\mathrm{P4}}$ & $\underline{\mathrm{T}}$ & $\underline{\mathrm{T} 6}$ & $\underline{\mathrm{O} 1}$ & $\underline{\mathrm{O} 2}$ & $\underline{\mathrm{Mean}}$ \\
\hline Sensitivity (SE) & 0.9667 & $\underline{0.9523}$ & $\underline{0.9531}$ & $\underline{0.9441}$ & 0.9562 & 0.947 & 0.9414 & 0.9632 & 0.94666 & 0.95117 \\
\hline Specificity (SP) & 0.9582 & 0.9616 & 0.9538 & 0.9443 & 0.9586 & 0.9621 & 0.9513 & 0.9641 & 0.9413 & 0.95302 \\
\hline Accuracy (ACC) & 0.9558 & 0.9669 & 0.953 & 0.9411 & 0.9588 & 0.9669 & 0.9552 & 0.9519 & 0.9448 & 0.95402 \\
\hline
\end{tabular}

Table 5. Sensitivity, Specificity and Accuracy metrics for CRQA Trapping Time for the experiments performed for both groups.

Finally, Table 6 shows SE, SP and AC for trend. In this table, it is shown that this is the RQA metric with the lower values. For instance, SE ranges fro, 0.8811 (T3 electrode) to 0.9148 (F8 electrode) with a mean of 0.9055. Also, SP shows a lower value of 0.882 (F8) and a higher value of $0.9164(\mathrm{Cz})$ with a mean of 0.8984 , whilst AC shows a lower value of 0.8834 (C4) and the higher value of 0.9154 (O1) with a mean of 0.9001 . This seems to indicate that although it is fairly constant, it falls short from the baseline values we aim to improve.

\begin{tabular}{|c|c|c|c|c|c|c|c|c|c|c|}
\hline Trend & $\underline{F z}$ & $\underline{\mathrm{Cz}}$ & $\underline{\mathrm{Pz}}$ & $\underline{\mathrm{C} 3}$ & T3 & C4 & T4 & Fp1 & $\mathrm{Fp} 2$ & F3 \\
\hline Sensitivity (SE) & 9113 & $0 . \overline{9136}$ & 8982 & 0.8836 & $0 . \overline{88}$ & $0 . \overline{90}$ & & & & 0.92 \\
\hline & & & & & & & & & & \\
\hline & & & & 0.85 & & & & & & \\
\hline & & $\mathrm{F}$ & & $\mathrm{P} 3$ & $\mathrm{P}$ & $\underline{\mathrm{T} 5}$ & $\mathrm{~T}$ & $\underline{\mathrm{o}}$ & $\underline{\mathrm{O} 2}$ & Mean \\
\hline & & & & & & & & & & \\
\hline & & & & & & & & & & \\
\hline$x(A C C)$ & 0.8956 & 0.8999 & 0.8947 & 0.9016 & 0.9001 & 0.9022 & 0.9049 & 0.9154 & 0.9104 & 0.90015 \\
\hline
\end{tabular}


Table 6. Sensitivity, Specificity and Accuracy metrics for CRQA Trend for the experiments performed for both groups.

Figure 4 shows Sensitivity for all metrics (Recurrence, Determinism, Entropy, Laminarity, Trapping Time, and Trend). In this figure it is shown in comparison with the value in the baseline shown by Mohammadi (2016), where all means with the exception for Trend are higher in the experiments shown in this contribution. Although Trend does not show a considerable low value, it may be discarded for sensitivity if a high classification want to be achieved using the proposed methodology.

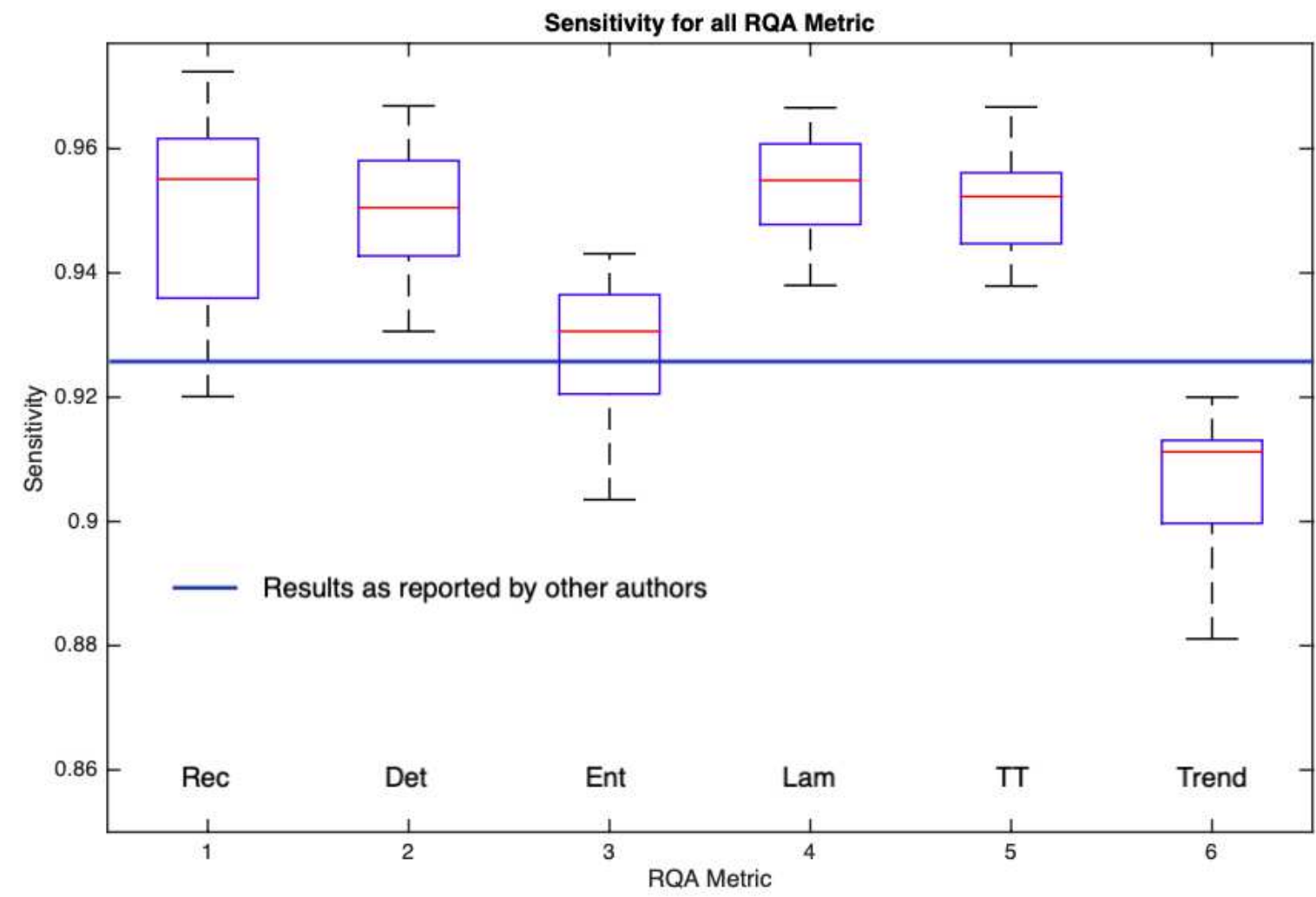

Figure 4. Sensitivity Quality Metric for each CRQA feature.

Figure 5 shows the specificity for all quality metrics. This figure shows similarities with sensitivity, in which Recurrence, Determinism, Laminarity and Trapping Time show high values, whereas Entropy shows slightly lower values and trend falls below the baseline. This also seem to indicate that given the right RQA features, a high classification rate to detect ADHD in EEG signal can be achieved. 
Lastly, Figure 6 shows the accuracy for all quality metrics. In this figure, it is shown that for most RQA feature, the accuracy is high with a few exceptions and most of the mean for these features are higher than the baseline (again, the exception being trend). This also seem to indicate that this methodology is not prone to detect false positives and show high rates of correct classifications when using the correct features. The experimental results indicate that RQA trend may not be used in order to achieve higher accuracy using the proposed methodology.

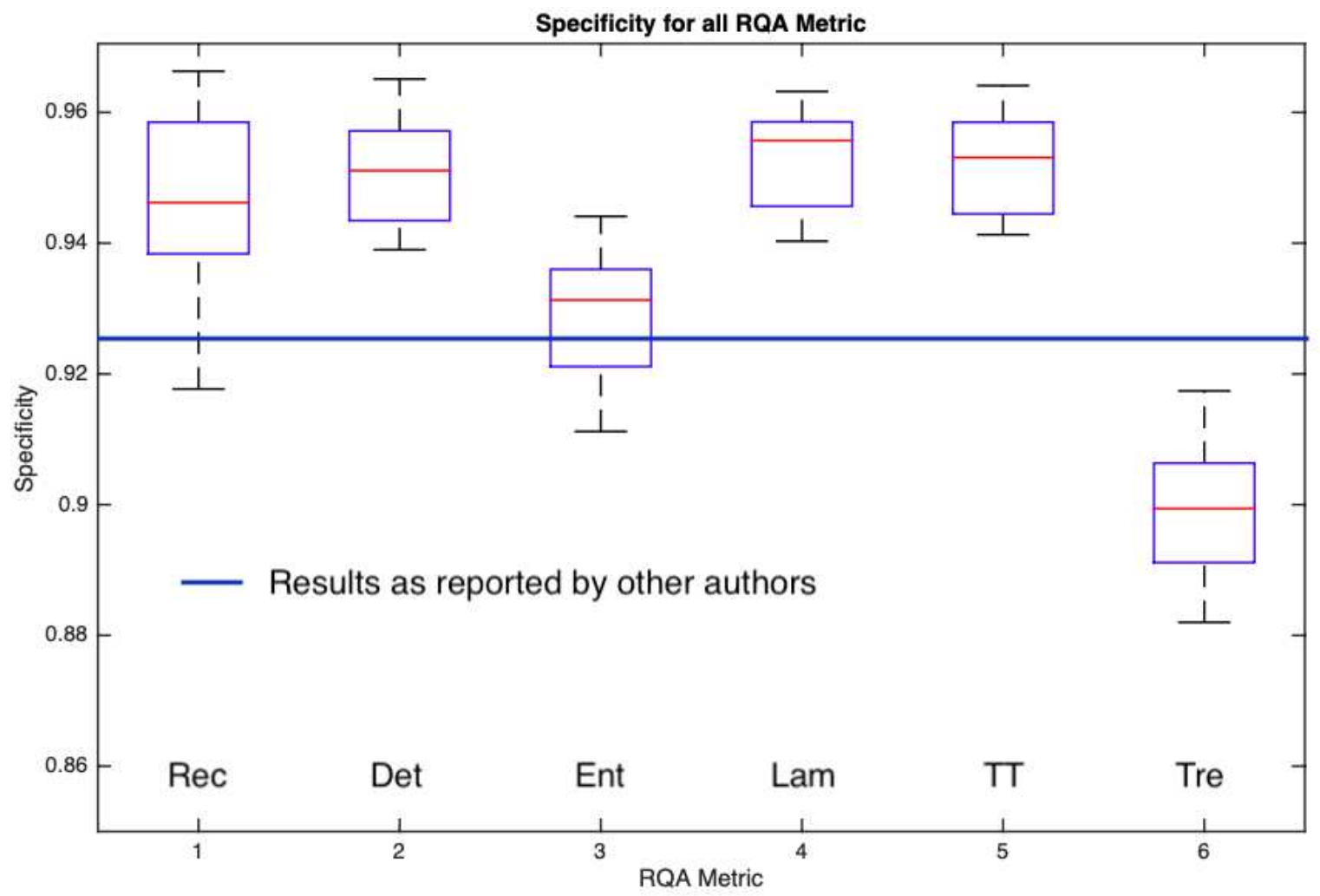

Figure 5. Specificity Quality Metric for each CRQA feature. 


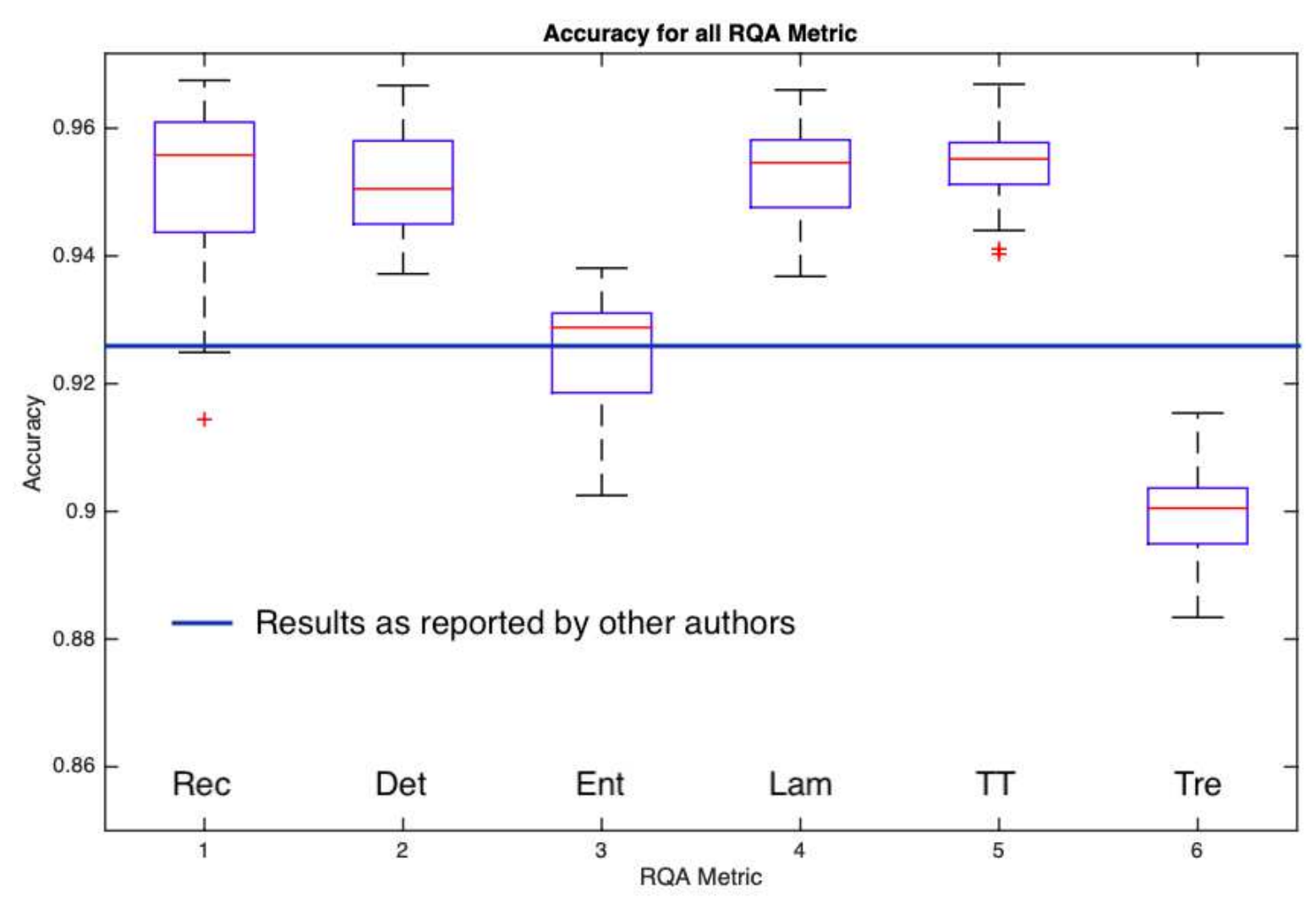

Figure 6. Accuracy Quality Metric for each CRQA feature.

\section{Conclusions and Future Work}

The proposed methodology shows that it is feasible to accurately classify ADHD using EEG signals, with a slight improvement upon other techniques used in the literature. Also, the reliability of this method is high as this is not prone to classify false positives. Some RQA feature prove to give better results than others, to the right tools must be selected in order to increase the classification rate.

Furthermore, this methodology presents many advantages with respect to existing machine learning algorithms. For instance, an effective training must be made in order to make an accurate classification for machine learning which is not required using the proposed methodology. Also, the classification rate is high regardless of the EEG electrode placed in the subject and does not need to discriminate some electrodes as shown on the experimental procedure.

Lastly, this work shows that the proposed methodology is not prone to detect false positives and false negative classifications, which is also a contribution of the present work. For future work, it may be worthwhile to explore the reason as to why trend shows a lower classification rate for sensitivity, specificity and accuracy.

\section{Conflict of Interest}

The authors declare that there is no conflict of interest at present regarding the publication of this paper. 


\section{Acknowledgements}

The authors would like to acknowledge the financial support of the Mexican government via The National Council of Science and Technology (CONACyT) funding.

\section{References}

[1] Aboofazeli M. and Z.K. Moussavi, Comparison of recurrence plot features of swallowing and breathe sounds CHAOS SOLITON FRACT, 2008 37:454-464.

[2] Aceves-Fernandez M.A., JC Pedraza-Ortega, A Sotomayor-Olmedo. (2014). Analysis of key features of non-linear behaviour using recurrence quantification. Case study: urban airborne pollution at Mexico City, Environmental Modeling \& Assessment 19 (2), 139-152.

[3] Aceves-Fernandez, M. A., Ramos-Arreguin, J. M., Gorrostieta-Hurtado, E., \& PedrazaOrtega, J. C. (2019). Methodology Proposal of EMG Hand Movement Classification Based on Cross Recurrence Plots. Computational and Mathematical Methods in Medicine, 2019.

[4] Addo P.M., M. Billio, and D. Guegan, Nonlinear Dynamics and Recurrence Plots for Detecting Financial Crisis. The North American Journal of Economics and Finance, 2013, 26, pp. 416-435.

[5] Aparicio T., Pozo, E. F., \& Saura, D. (2008). Detecting determinism using recurrence quantification analysis: Three test procedures. Journal of Economic Behavior E Organization, 65(3-4), 768-787.

[6] Barbé, K., Pintelon, R., \& Schoukens, J. (2009). Welch method revisited: nonparametric power spectrum estimation via circular overlap. IEEE Transactions on signal processing, 58(2), 553565.

[7] Bastos J.A. and J. Caiado, Recurrence quantification analysis of global stock markets, PHYSICA A, 390 (2011) 1315-1325.

[8] Becker, K., Schneider, G., Eder, M., Ranft, A., Kochs, E. F., Zieglgänsberger, W., \& Dodt, H. U. (2010). Anaesthesia monitoring by recurrence quantification analysis of EEG data. PloS one, 5(1), e8876.

[9] Bradley E. and R. Mantilla, Recurrence plots and unstable periodic orbits. Chaos. 2002, 123:596-600. [42] N. Marwan, M. Thiel, and N. R. Nowaczyk, Cross recurrence plot based synchronization of time series, NONLINEAR PROC GEOPH, (2002) 9: 325-331.

[10]Demos, A. P., \& Chaffin, R. (2017). Removing obstacles to the analysis of movement in musical performance: Recurrence, mixed models, and surrogates. The Routledge companion to embodied music interaction. New York, NY: Routledge.

[11]Dose, H., Møller, J. S., Iversen, H. K., \& Puthusserypady, S. (2018). An end-to-end deep learning approach to MI-EEG signal classification for BCIs. Expert Systems with Applications, $114,532-542$.

[12] Eckmann, J. P., Kamphorst, S. O., \& Ruelle, D. (1987). Recurrence plots of dynamical systems. EPL (Europhysics Letters), 4(9), 973.

[13] Elias J., \& Namboothiri, V. N. (2014). Cross-recurrence plot quantification analysis of input and output signals for the detection of chatter in turning. Nonlinear Dynamics, 76(1), 255261.

[14] Eslami, T., \& Saeed, F. (2018, May). Similarity based classification of ADHD using singular value decomposition. In Proceedings of the 15th ACM International Conference on Computing Frontiers (pp. 19-25).

[15] Felt, B. T., Biermann, B., Christner, J. G., Kochhar, P., \& Van Harrison, R. (2014). Diagnosis and management of ADHD in children. American Family Physician, 90(7), 456-464.

[16]Fernandez-Fraga, S. M., Aceves-Fernandez, M. A., Rodríguez-Resendíz, J., Pedraza-Ortega, J. C., \& Ramos-Arreguín, J. M. (2019). Steady-state visual evoked potential (SSEVP) from EEG signal modeling based upon recurrence plots. Evolving Systems, 10(2), 97-109.

[17] Gao, J., \& Cai, H. (2000). On the structures and quantification of recurrence plots. Physics Letters A, 270(1-2), 75-87. 
[18] Ghassemi, F., Hassan_Moradi, M., Tehrani-Doost, M., \& Abootalebi, V. (2012). Using nonlinear features of EEG for ADHD/normal participants' classification. Procedia-Social and Behavioral Sciences, 32, 148-152.

[19] Goswami B., G. Ambika, N. Marwan, On interrelations of recurrences and connectivity trends between stock indices, PHYSICA A, 391 (2012) 4364-4376.

[20] Huke, J. P. (2006). Embedding nonlinear dynamical systems: A guide to Takens' theorem.

[21] Khodabakhshi, M. B., \& Saba, V. (2020). A nonlinear dynamical approach to analysis of emotions using EEG signals based on the Poincaré map function and recurrence plots. Biomedical Engineering/Biomedizinische Technik, 1(ahead-of-print).

[22] Kuang, D., \& He, L. (2014, November). Classification on ADHD with deep learning. In 2014 International Conference on Cloud Computing and Big Data (pp. 27-32). IEEE.

[23]Kumar, S., Sharma, A., Mamun, K., \& Tsunoda, T. (2016, December). A deep learning approach for motor imagery EEG signal classification. In 2016 3rd Asia-Pacific World Congress on Computer Science and Engineering (APWC on CSE) (pp. 34-39). IEEE.

[24] Litak G. and R. Rusinek, Dynamics of a stainless steel turning process by statistical and recurrence analyses, Meccanica (2012) 47:1517-1526.

[25]Litak, G., Kecik, K., \& Rusinek, R. (2013). Cutting force response in milling of Inconel: Analysis by wavelet and Hilbert-Huang transforms. Latin American Journal of Solids and Structures, 10(1), 133-140.

[26] March, T. K., Chapman, S. C., \& Dendy, R. O. (2005). Recurrence plot statistics and the effect of embedding. Physica D: Nonlinear Phenomena, 200(1-2), 171-184.

[27] Marwan, N., \& Kurths, J. (2004). Cross recurrence plots and their applications. Mathematical physics research at the cutting edge, 101-139.

[28] Marwan, N., Romano, M. C., Thiel, M., \& Kurths, J. (2007). Recurrence plots for the analysis of complex systems. Physics reports, 438(5-6), 237-329.

[29] Marwan, N., Schinkel, S., \& Kurths, J. (2013). Recurrence plots 25 years later-gaining confidence in dynamical transitions. EPL (Europhysics Letters), 101(2), 20007.

[30] Mohammadi, M. R., Khaleghi, A., Nasrabadi, A. M., Rafieivand, S., Begol, M., \& Zarafshan, H. (2016). EEG classification of ADHD and normal children using non-linear features and neural network. Biomedical Engineering Letters, 6(2), 66-73.

[31] Mueller, A., Candrian, G., Kropotov, J. D., Ponomarev, V. A., \& Baschera, G. M. (2010, June). Classification of ADHD patients on the basis of independent ERP components using a machine learning system. In Nonlinear biomedical physics (Vol. 4, No. S1, p. S1). BioMed Central.

[32] Nagabushanam, P., George, S. T., \& Radha, S. (2019). EEG signal classification using LSTM and improved neural network algorithms. Soft Computing, 1-23.

[33] Nalband, S., Sundar, A., Prince, A. A., \& Agarwal, A. (2016). Feature selection and classification methodology for the detection of knee-joint disorders. Computer methods and programs in biomedicine, 127, 94-104.

[34] Ngamga E.J., S. Bialonski, N. Marwan, Evaluation of selected recurrence measures in discriminating pre-ictal and inter-ictal periods from epileptic EEG data, PHYS LETT A, 380 (2016) 1419-1425.

[35] Peng, X., Lin, P., Zhang, T., \& Wang, J. (2013). Extreme learning machine-based classification of ADHD using brain structural MRI data. PloS one, 8(11), e79476.

[36] Popescu, F., Enache, F., Vizitiu, I. C., \& Ciotirnae, P. (2014, May). Recurrence plot analysis for characterization of appliance load signature. In Communications (COMM), 2014 10th International Conference on (pp. 1-4). IEEE.

[37] Rahi, P. K., \& Mehra, R. (2014). Analysis of power spectrum estimation using welch method for various window techniques. International Journal of Emerging Technologies and Engineering, 2(6), 106-109.

[38] Rashvandi Z, A.M. Nasrabadi, Classification of hypnotisable groups based on normal EEG signals using the Recurrence Quantification Analysis and Support Vector Machine, 23rd. Iranian Conference on Electrical Engineering, IEEE, ISBN: 978-1-4799-1972-7, 2015. 
[39] Rau, S., Skapek, M. F., Tiplady, K., Seese, S., Burns, A., Armour, A. C., \& Kenworthy, L. (2020). Identifying comorbid ADHD in autism: Attending to the inattentive presentation. Research in Autism Spectrum Disorders, 69, 101468.

[40] Richhariya, B., \& Tanveer, M. (2018). EEG signal classification using universum support vector machine. Expert Systems with Applications, 106, 169-182.

[41] Sadatnezhad, K., Boostani, R., \& Ghanizadeh, A. (2011). Classification of BMD and ADHD patients using their EEG signals. Expert Systems with Applications, 38(3), 1956-1963.

[42] Satapathy, S. K., Dehuri, S., \& Jagadev, A. K. (2017). EEG signal classification using PSO trained RBF neural network for epilepsy identification. Informatics in Medicine Unlocked, 6, 111.

[43] Samavati, M., Nasrabadi, A. M., \& Mohammadi, M. R. (2012, May). Automatic minimization of eye blink artifacts using fractal dimension of independent components of multichannel EEG. In 20th Iranian Conference on Electrical Engineering (ICEE2012) (pp. 1576-1578). IEEE.

[44] Silva L., Vaz, J. R., Castro, M. A., Serranho, P., Cabri, J., \& Pezarat-Correia, P. (2015). Recurrence quantification analysis and support vector machines for golf handicap and low back pain EMG classification. Journal of Electromyography and Kinesiology, 25(4), 637-647.

[45] Strozzi F., Zaldívar, J. M., \& Zbilut, J. P. (2007). Recurrence quantification analysis and state space divergence reconstruction for financial time series analysis. Physica A: statistical mechanics and its applications, 376, 487-499.

[46]Subasi, A., \& Ercelebi, E. (2005). Classification of EEG signals using neural network and logistic regression. Computer methods and programs in biomedicine, 78(2), 87-99.

[47] Tenev, A., Markovska-Simoska, S., Kocarev, L., Pop-Jordanov, J., Müller, A., \& Candrian, G. (2014). Machine learning approach for classification of ADHD adults. International Journal of Psychophysiology, 93(1), 162-166.

[48] Torse, D. A., Khanai, R., \& Desai, V. V. (2019, April). Classification of Epileptic Seizures using Recurrence Plots and Machine Learning Techniques. In 2019 International Conference on Communication and Signal Processing (ICCSP) (pp. 0611-0615). IEEE.

[49] Welch P., "The use of fast fourier transform for the estimation of power spectra: A method based on time averaging over short, modified peri-odograms," IEEE Trans. Audio Electroacoust., vol. AE-15, pp. 70-73, Jun. 1967.

[50] Yulmetyev, R. M., \& Gafarov, F. M. (1999). Dynamics of the information entropy in random processes. Physica A: Statistical Mechanics and its Applications, 273(3-4), 416-438.

[51]Zhou, D., \& Li, X. (2020). Epilepsy EEG signal classification algorithm based on Improved RBF. Frontiers in Neuroscience.

[52]Zou Y., R.V. Donner, J.F. Donges, Identifying complex periodic windows in continuous-time dynamical systems using recurrence-based methods, Chaos. 20, 2010. 


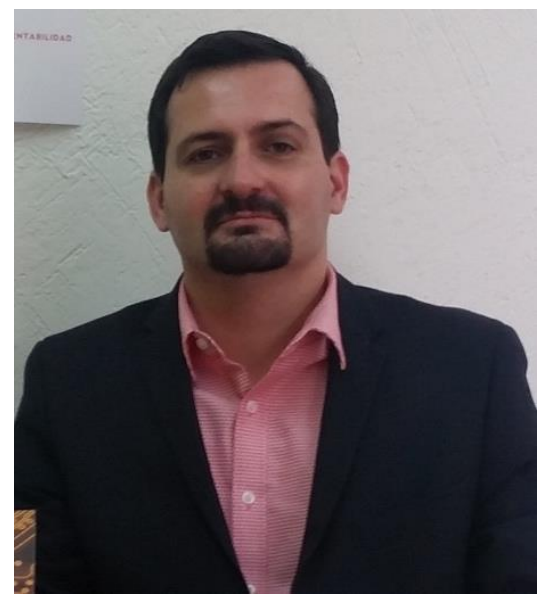

Marco A. Aceves-Fernandez obtained his B.Sc. (Eng) in Telematics at the Universidad de Colima, Mexico. He obtained both his M.Sc. and Ph.D. at the University of Liverpool, England in the field of Intelligent Systems. He is a full professor at the Universidad Autonoma de Queretaro, Mexico. His interests include Embedded and Intelligent Systems. 


\section{Figures}

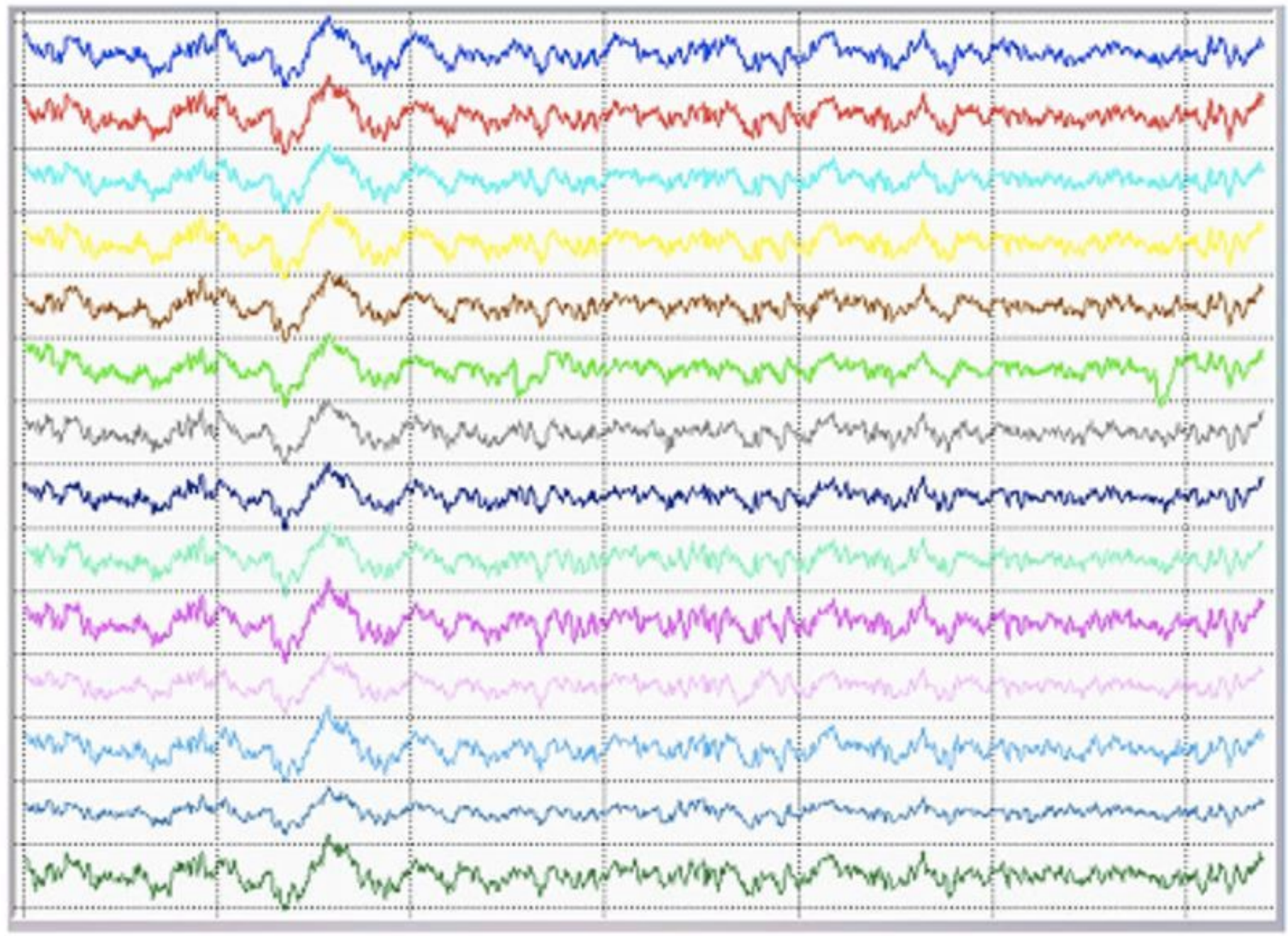

\section{Figure 1}

Set of signals derived from an electroencephalogram (EEG). 14 channels, sampling for $6.5 \mathrm{~s}$ (FernandezFraga, 2019) 


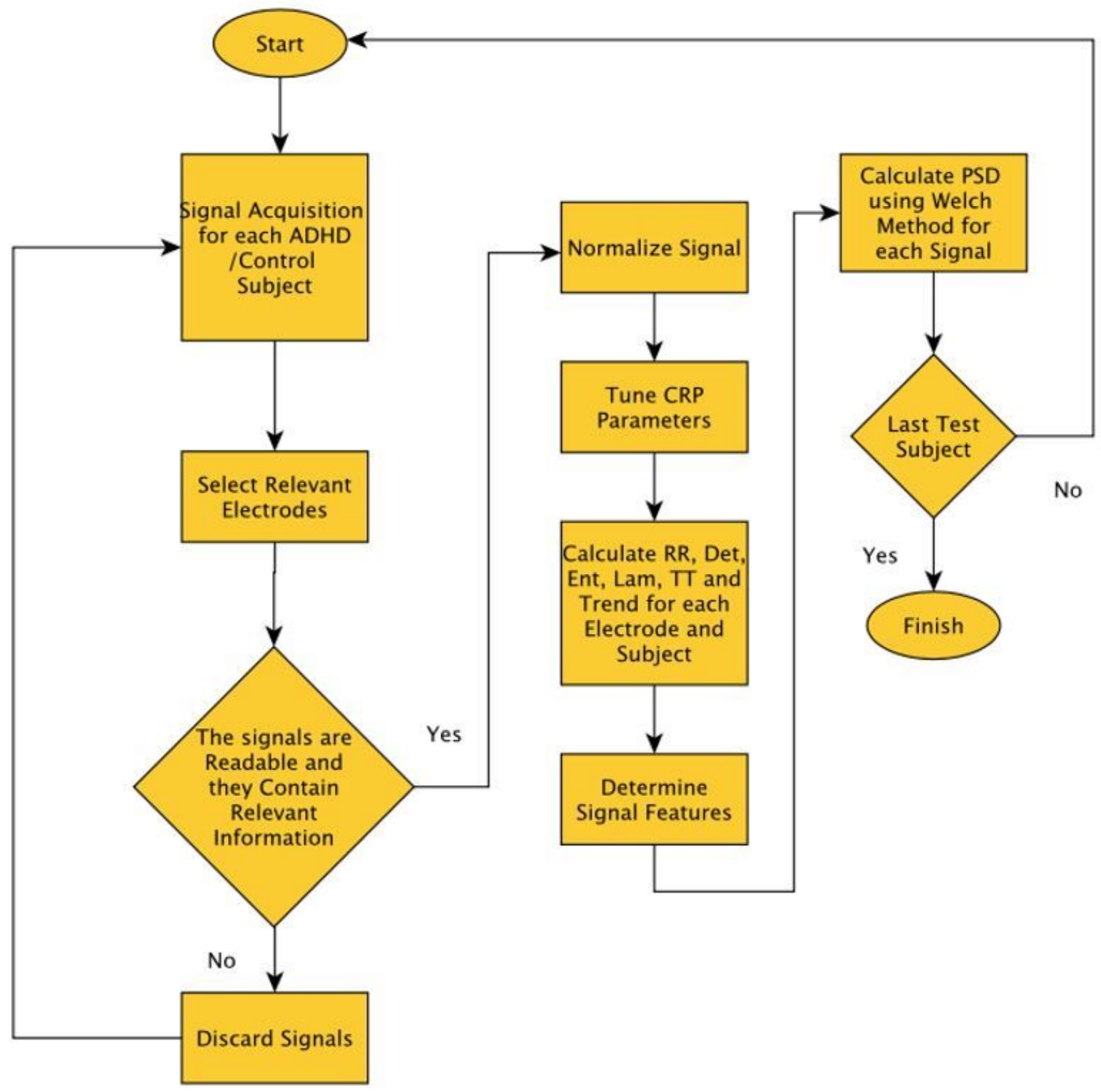

Figure 2

Methodology used in the present work. 


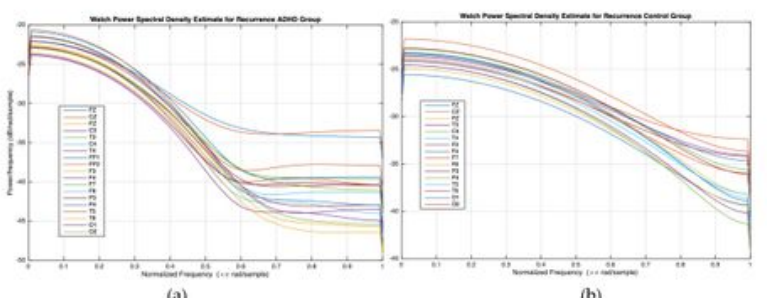

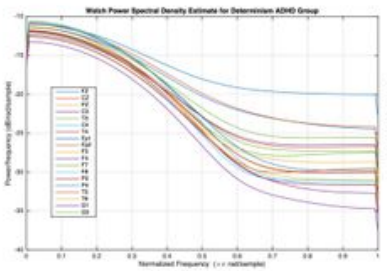

(c)

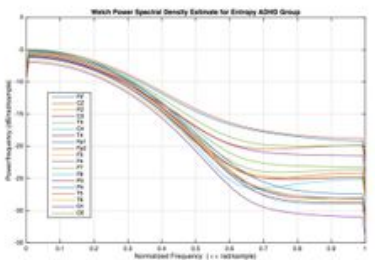

(e)

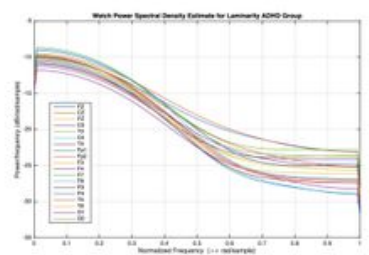

(g)

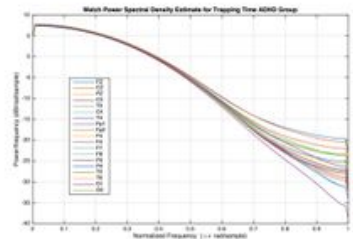

(i)

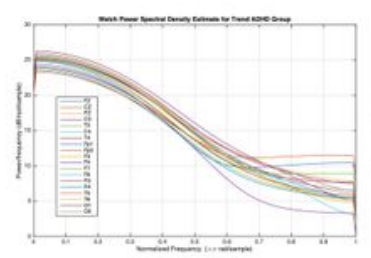

(k)

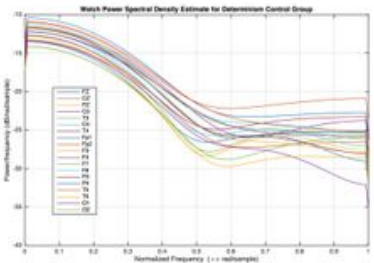

(d)

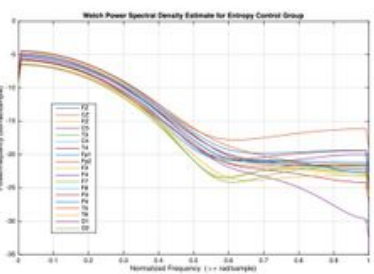

(f)

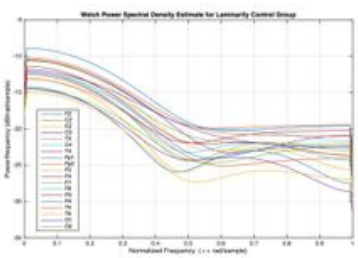

(h)

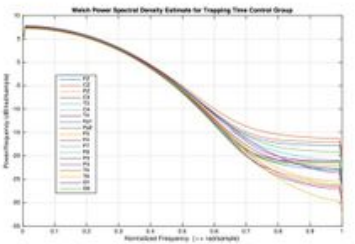

(j)

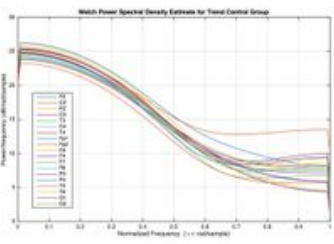

(1)

\section{Figure 3}

Results of RQA Measures using the Welch Power Spectral Density Method. a) Recurrence rate vs Power Frequency for ADHD Group, b) Recurrence rate vs Power Frequency for Control Group, c) Determinism vs Power Frequency for ADHD Group, d) Determinism vs Power Frequency for Control Group, e) Entropy vs Power Frequency for ADHD Group, f) Entropy vs Power Frequency for Control Group g) Laminarity vs Power Frequency for ADHD Group, h) Laminarity vs Power Frequency for Control Group, i) Trapping Time 
vs Power Frequency for ADHD Group, j) Trapping Time vs Power Frequency for Control Group, k) Trend vs Power Frequency for ADHD Group and I) Trend vs Power Frequency for Control Group.

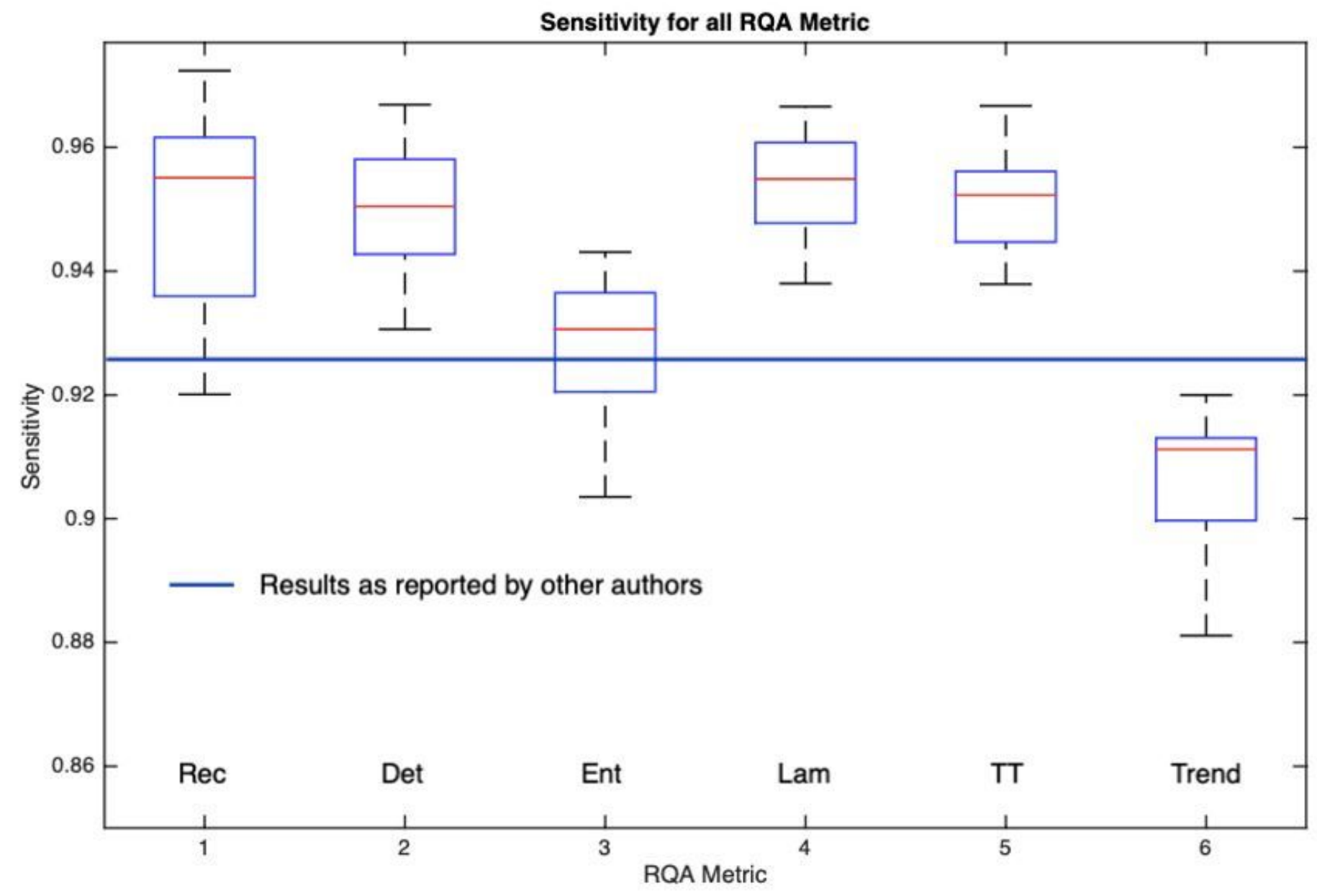

Figure 4

Sensitivity Quality Metric for each CRQA feature. 


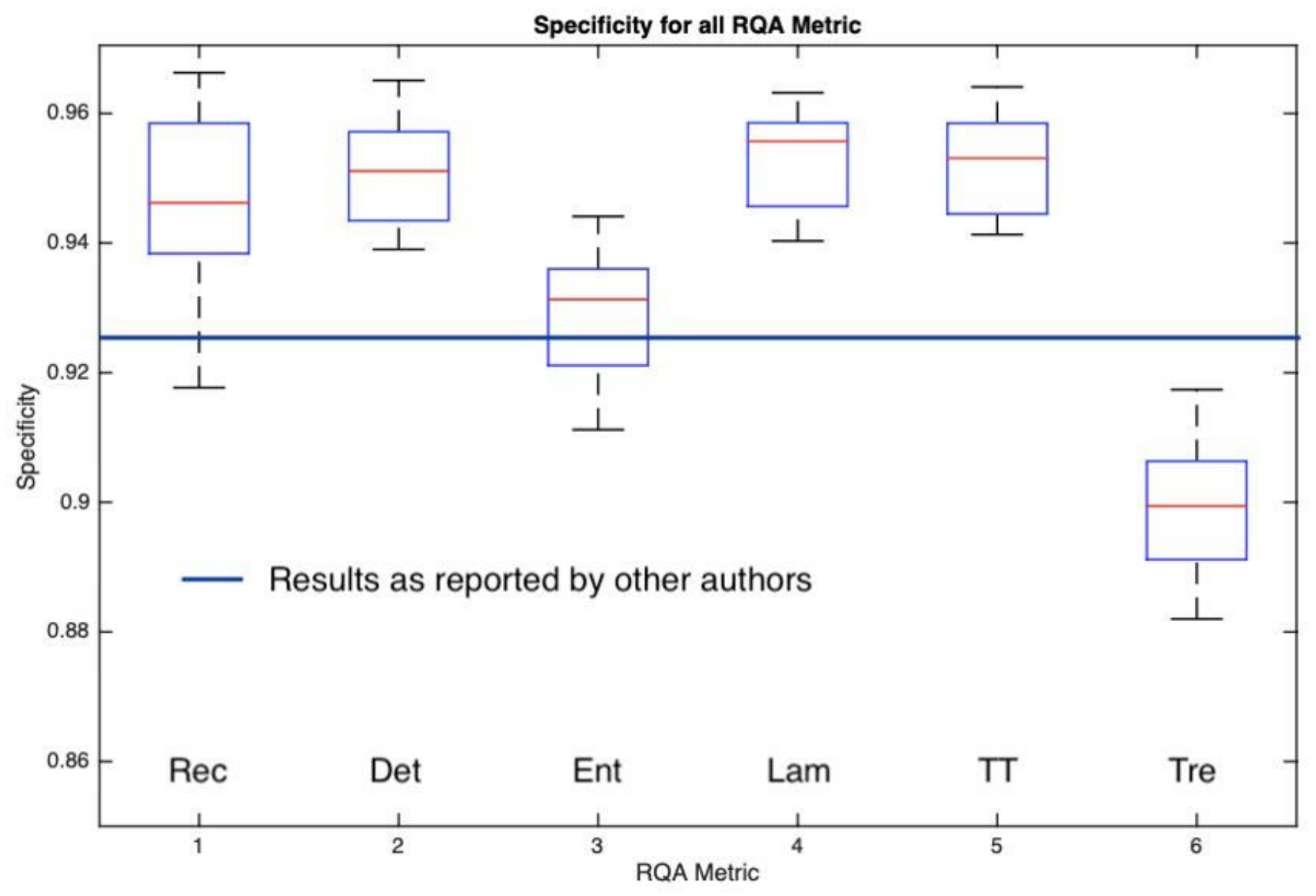

Figure 5

Specificity Quality Metric for each CRQA feature. 


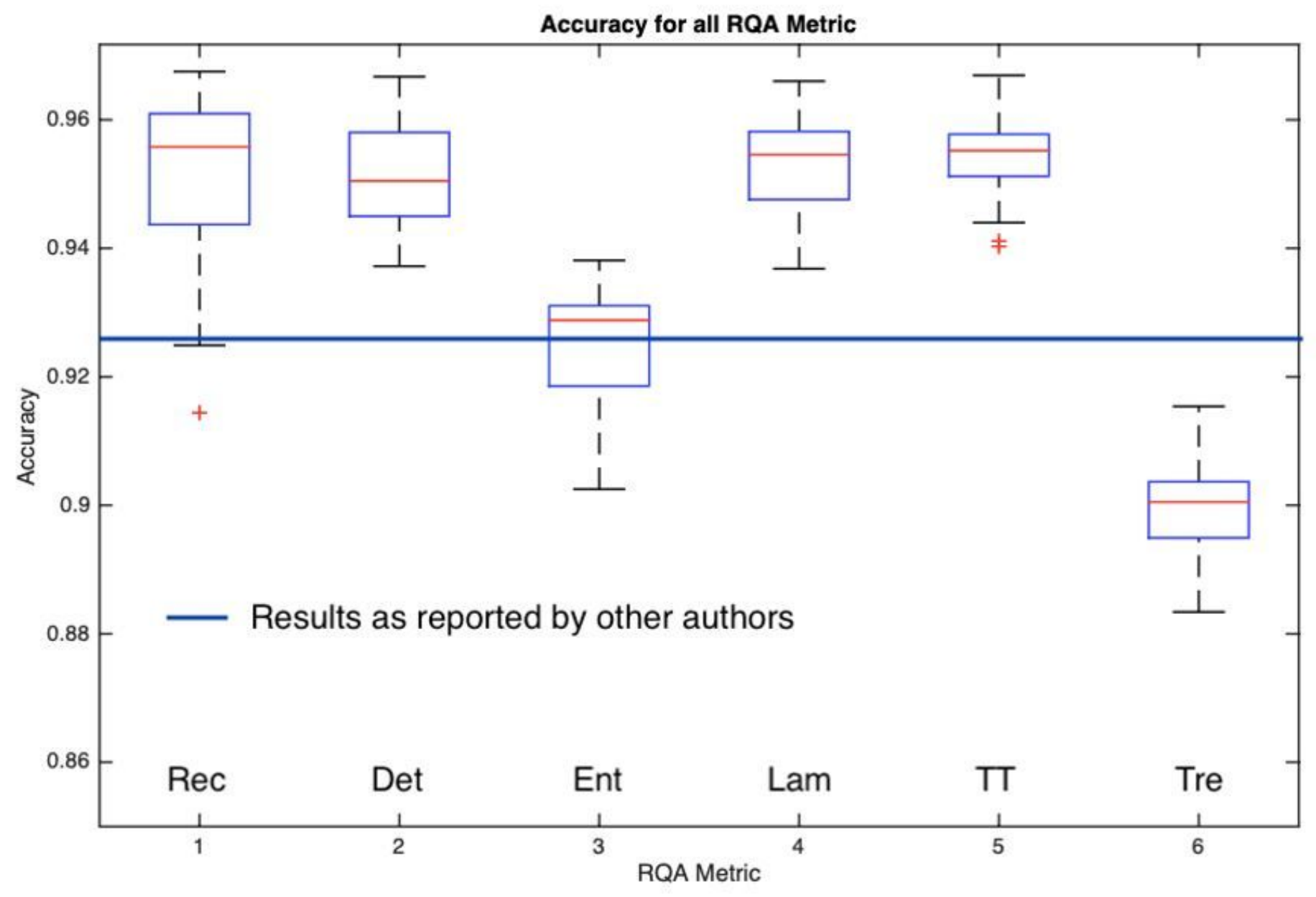

Figure 6

Accuracy Quality Metric for each CRQA feature. 